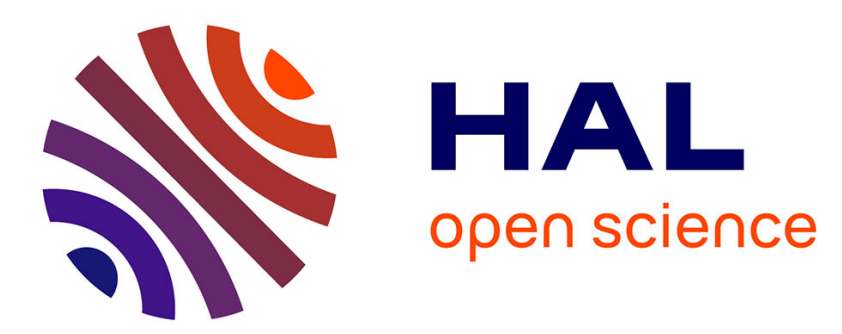

\title{
Advances in observer design for Takagi-Sugeno systems with unmeasurable premise variables
}

Dalil Ichalal, Benoît Marx, José Ragot, Didier Maquin

\section{To cite this version:}

Dalil Ichalal, Benoît Marx, José Ragot, Didier Maquin. Advances in observer design for TakagiSugeno systems with unmeasurable premise variables. 20th Mediterranean Conference on Control and Automation, MED 2012, Jul 2012, Barcelone, Spain. pp.848-853, 10.1109/MED.2012.6265744 . hal-00684701

\section{HAL Id: hal-00684701 https://hal.science/hal-00684701}

Submitted on 8 Apr 2014

HAL is a multi-disciplinary open access archive for the deposit and dissemination of scientific research documents, whether they are published or not. The documents may come from teaching and research institutions in France or abroad, or from public or private research centers.
L'archive ouverte pluridisciplinaire HAL, est destinée au dépôt et à la diffusion de documents scientifiques de niveau recherche, publiés ou non, émanant des établissements d'enseignement et de recherche français ou étrangers, des laboratoires publics ou privés. 


\title{
Advances in observer design for Takagi-Sugeno systems with unmeasurable premise variables
}

\author{
Dalil Ichalal, Benoît Marx, José Ragot, Didier Maquin
}

\begin{abstract}
This paper proposes a new approach of observer design for nonlinear systems described by a Takagi-Sugeno model. Its main contribution concerns models with premise variables depending on the system states which are completely or partially unknown. This case is more difficult than when the premise variables are known or measured. Indeed, in this case, weighting functions of the observer depend on state estimates and the state estimation error is then governed by a Lipschitz nonlinear system. Here, two main results are established. Firstly, relaxed stability conditions are provided, using a nonquadratic Lyapunov function, to guarantee asymptotic stability of the observer. This aims to reduce the conservativeness compared to the existing works and enhance the maximal admissible Lipschitz constant for which the linear matrix inequality (LMI) conditions are feasible. Secondly, the Input-to-State Stability concept combined to a nonquadratic Lyapunov function are used to guarantee a bounded state estimation error which relaxes the conservativeness related to the Lipschitz constant. The robustness aspect is dealt with respect to some bounded modeling uncertainties and additive bounded perturbations. The stability conditions are expressed in terms of LMI.
\end{abstract}

Index Terms-Nonlinear systems, Takagi-Sugeno systems, unmeasurable premise variables, LMI, observer design, Inputto-state stability.

\section{INTRODUCTION}

Observer design for nonlinear systems is a challenging problem which is intensively studied in control and diagnosis fields. Indeed, many approaches have been proposed in order to estimate the states of such systems. Firstly, in [30], the author proposed a method for systems modeled by a linear part and a nonlinear Lipschitz one for which stability conditions were proposed. Thereafter, in [23], an iterative algorithm was proposed to deal with the problem of observer design, however the method may fail even if the system is observable. In [24], the $H_{\infty}$ formulation is exploited in order to provide necessary and sufficient conditions for stability of the state estimation error for Lipschitz systems. This work is extended in [22] for dynamic gain observer design for the same class of systems. Many other works were also proposed, namely, sliding mode observers which are interesting due to their insensitivity to modeling uncertainties and external perturbations [6], or high gain observer which are based on a mathematical transformation of the system by using immersion techniques [7], [8].

D. Ichalal is with the IBISC laboratory, Université d'Evry-Vald'Essonne, 40, rue du Pelvoux, Courcouronnes 91020 Evry Cedex, France \{dalil.ichalal\}eibisc.univ-evry.fr

B. Marx, J. Ragot and D. Maquin are with the Centre de Recherche en Automatique de Nancy, Université de Lorraine - CNRS, 2, avenue de la forêt de Haye 54516 Vandoeuvre-les-Nancy, France \{benoit.marx, jose.ragot, didier.maquin\}@univ-lorraine.fr
In these last years, the Takagi-Sugeno (T-S) approach, introduced in [27], offered an interesting tool for studying nonlinear systems. This structure may represent or approximate strongly nonlinear systems with a simple mathematical representation consisting in several linear submodels weighted by nonlinear functions satisfying the convex sum property. These weighing functions may depend on measurable premise variables (input, output of the system or external variable as linear parameter variable (LPV) systems), or/and unmeasurable premise variables (UPV) as the state of the system. The T-S approach is interesting because it allows to extend some control and observation methods, previously dedicated to linear systems, to nonlinear ones [29].

The problem of state estimation of nonlinear systems using T-S model approach has been addressed with different methods, the most of the published works considered T-S models with measurable premise variables [1], [2], [17], [21]. Clearly the case of measurable premise variables offers a simpler way to generalize the methods already developed for linear systems. However, T-S models with UPV naturally arise when they are obtained from a nonlinear system $(\dot{x}=$ $f(x, u)$ ) by mathematical transformations. The most wellknown is the so-called sector nonlinearity transformation providing an exact T-S model with no loss of information, in a compact set of the state space. Furthermore, the T-S models with UPV may represent a larger class of nonlinear systems compared to the T-S model with measurable premise variables [32].

However, T-S models with UPV are more difficult to deal with than those with measurable premise variables. Consequently, few works are devoted to this class of models despite of their advantages. Nevertheless, we can cite [5] which is the first work dealing with the problem of observer design for this class of nonlinear systems. Extensions of this approach have also been published in [4], [15] and [20]. Let us also mention [32] where a filter estimating the state and minimizing the effect of disturbances is proposed. More recently, new approaches have been proposed in [10], [11], [12], [13]. In [10], the differential mean value theorem is combined to sector nonlinearity transformation to re-write the state estimation error as an autonomous T-S system in order to be able to apply the classical stability studies developed for T-S systems. A similar problem is studied recently for a special case of discrete-time LPV systems with uncertain parameters in [9] and [18].

In this work, observer design approaches are proposed for estimating the state of nonlinear systems represented by a T$\mathrm{S}$ model with UPV. The contributions of this paper concern: 
the establishing of relaxed stability conditions by the use of a nonquadratic Lyapunov function, the increasing of the maximal admissible Lipschitz constant allowing to obtain a solution and finally, the combination of nonquadratic Lyapunov function with input-to-state stability (ISS) to ensure the bounded state estimation error which is used to deal with T-S systems with modeling uncertainties. The established inequalities are stated in an adequate form for applying several relaxations provided in the literature as Polya's theorem [25], Tuan's lemma [31], or the use of nonquadratic Lyapunov functions [28]. The paper is organized as follows: In section II, an observer ensuring asymptotic convergence is proposed by using a nonquadratic Lyapunov function and the Lipschitz condition. In section III, an approach combining nonquadratic Lyapunov function and ISS concept is proposed in order to overcome the problem related to the Lipschitz constant and finally, in the last section, conclusion and future works are discussed.

\section{ASYMPTOTIC OBSERVER DESIGN}

Let us consider a nonlinear system described by the following T-S model

$$
\left\{\begin{aligned}
\dot{x}(t) & =\sum_{i=1}^{r} \mu_{i}(x(t))\left(A_{i} x(t)+B_{i} u(t)\right) \\
y(t) & =C x(t)
\end{aligned}\right.
$$

where $x(t) \in \mathbb{R}^{n}$ is the state vector, $u(t) \in \mathbb{R}^{n_{u}}$ is the input vector and $y(t) \in \mathbb{R}^{n_{y}}$ represents the output vector. $A_{i} \in$ $\mathbb{R}^{n \times n}, B_{i} \in \mathbb{R}^{n \times n_{y}}, C_{i} \in \mathbb{R}^{n_{y} \times n}$ are known matrices and $r$ is the number of sub-models. The functions $\mu_{i}(x(t))$ are the weighting functions depending on the state variables $x(t)$ which are non measurable variables. These functions verify the so-called convex sum properties

$$
\sum_{i=1}^{r} \mu_{i}(x(t))=1, \quad 0 \leq \mu_{i}(x(t)) \leq 1 \quad \forall i \in\{1, \ldots, r\}
$$

The output equation is chosen to be linear with regard the state, which is frequently the case in practice.

\section{A. Observer structure}

Let us first introduce the following notations

$$
\begin{aligned}
& X_{\mu}=\sum_{i=1}^{r} \mu_{i}(x(t)) X_{i}, X_{\mu \mu}=\sum_{i=1}^{r} \sum_{j=1}^{r} \mu_{i}(x(t)) \mu_{j}(x(t)) X_{i j} \\
& X_{\hat{\mu}}=\sum_{i=1}^{r} \hat{\mu}_{i}(x(t)) X_{i}, X_{\hat{\mu} \hat{\mu}}=\sum_{i=1}^{r} \sum_{j=1}^{r} \hat{\mu}_{i}(x(t)) \hat{\mu}_{j}(x(t)) X_{i j}
\end{aligned}
$$

where $X_{i}$ and $X_{i j}$ stands for any matrices, e.g. $A_{i}$ or $B_{i}$. Using these notations, the system (1) becomes

$$
\left\{\begin{array}{l}
\dot{x}(t)=A_{\mu} x(t)+B_{\mu} u(t) \\
y(t)=C x(t)
\end{array}\right.
$$

The proposed observer is given by

$$
\left\{\begin{array}{l}
\dot{\hat{x}}(t)=A_{\hat{\mu}} \hat{x}(t)+B_{\hat{\mu}} u(t)+P_{\hat{\mu}}^{-1} L_{\hat{\mu}}(y(t)-\hat{y}(t)) \\
\hat{y}(t)=C \hat{x}(t)
\end{array}\right.
$$

where the matrices $L_{i}$ and the symmetric positive definite matrices $P_{i}$ are to be determined. The state estimation error $e(t)=x(t)-\hat{x}(t)$ is governed by the following nonlinear differential equation depending on the system state $x(t)$, its estimate $\hat{x}(t)$, the system input $u(t)$ and the state estimation error $e(t)$

$$
\dot{e}(t)=\Phi_{\hat{\mu} \hat{\mu}} e(t)+\delta(x, \hat{x}, u)
$$

where

$$
\begin{aligned}
\Phi_{\hat{\mu} \hat{\mu}} & =A_{\hat{\mu}}-P_{\hat{\mu}}^{-1} L_{\hat{\mu}} C \\
\delta(x, \hat{x}, u) & =f(\hat{x}, x, u)-f(x, x, u) \\
f(\hat{x}, x, u) & =A_{\hat{\mu}} x(t)+B_{\hat{\mu}} u(t)
\end{aligned}
$$

with $P_{i}=P_{i}^{T}>0$. In this section, the following assumptions are made

- A1. The function $f$ in (10) is Lipschitz with respect to its first variable. Then, there exists a positive scalar $\eta$ such that $\delta^{T}(x, \hat{x}, u) \delta(x, \hat{x}, u) \leq \eta^{2} e^{T}(t) e(t)$.

- A2. There exists positive scalars $\rho_{i}$ such that the weighting functions satisfy $\mid \dot{\mu}_{i}\left(\hat{x}(t) \mid \leq \rho_{i}\right.$.

It is not always necessary to check the assumption A2 a posteriori, since the definition of the functions $\mu_{i}$ may ensure this property for any value of $\hat{x}(t)$.

\section{B. Stability analysis}

First sufficient LMI conditions for asymptotic convergence of the state estimation error (7) are given in the next theorem.

Theorem 1: Under the assumptions $\mathbf{A 1}$ and $\mathbf{A 2}$, if there exists a symmetric matrix $P_{0}$, symmetric and positive definite matrices $P_{i}$, gain matrices $L_{i}$ and a positive scalar $\lambda$ satisfying the following LMI

$$
\begin{aligned}
M_{i j} & <0, i, j=1, \ldots, r \\
P_{i}-P_{0} & \geq 0, i=1, \ldots, r
\end{aligned}
$$

where

$$
M_{i j}=\left(\begin{array}{cc}
\Omega_{i j} & P_{j} \\
P_{j} & -\lambda I
\end{array}\right)
$$

and

$$
\Omega_{i j}=A_{i}^{T} P_{j}+P_{j} A_{i}-C^{T} L_{i}^{T}-L_{i} C+\sum_{i=1}^{r} \rho_{i}\left(P_{i}-P_{0}\right)+\lambda \eta^{2} I
$$

then the state estimation error asymptotically converges towards zero.

Proof: To prove the stability of the state estimation error (7), let us consider the nonquadratic Lyapunov function

$$
V(e(t))=e^{T}(t) P_{\hat{\mu}} e(t)
$$

The matrices $P_{i}$ being symmetric positive definite and the weighting functions satisfying (2), the function $V(e(t))$ is positive too. Its time derivative is given by

$$
\dot{V}(e(t))=\dot{e}^{T}(t) P_{\hat{\mu}} e(t)+e^{T}(t) P_{\hat{\mu}} \dot{e}(t)+e^{T}(t) \dot{P}_{\hat{\mu}} e(t)
$$

The derivative of $P_{\hat{\mu}}$ is given by

$$
\dot{P}_{\hat{\mu}}=\sum_{i=1}^{r} \dot{\mu}_{i}(\hat{x}) P_{i}
$$


Substituting $\dot{P}_{\hat{\mu}}$ and (7) in (16), one obtains

$$
\begin{aligned}
\dot{V}(e(t)) & =e^{T}(t)\left(\Phi_{\hat{\mu} \hat{\mu}}^{T} P_{\hat{\mu}}+P_{\hat{\mu}} \Phi_{\hat{\mu} \hat{\mu}}+\dot{P}_{\hat{\mu}}\right) e(t) \\
& +2 e^{T}(t) P_{\hat{\mu}} \delta(x, \hat{x}, u)
\end{aligned}
$$

With assumption A1 and since it is well known that the following inequality holds

$$
X^{T} Y+Y^{T} X \leq X^{T} \Sigma X+Y^{T} \Sigma^{-1} Y
$$

for any matrices $X, Y$ and $\Sigma=\Sigma^{T}>0$, then, for any positive $\lambda$, it follows

$$
2 e^{T}(t) P_{\hat{\mu}} \delta(x, \hat{x}, u) \leq \lambda \eta^{2} e^{T}(t) e(t)+\lambda^{-1} e^{T}(t) P_{\hat{\mu}} P_{\hat{\mu}} e(t)
$$

Due to (2), it obviously follows

$$
\sum_{i=1}^{r} \dot{\mu}_{i}(\hat{x})=0 \Rightarrow \sum_{i=1}^{r} \dot{\mu}_{i}(\hat{x}) P_{0}=0
$$

with assumption $\mathbf{A} 2$ and (21), $\dot{P}_{\hat{\mu}}$ is bounded by

$\dot{P}_{\hat{\mu}}=\sum_{i=1}^{r} \dot{\mu}_{i}(\hat{x})\left(P_{i}-P_{0}\right) \leq \sum_{i=1}^{r}\left|\dot{\mu}_{i}(\hat{x})\right|\left(P_{i}-P_{0}\right)=\sum_{i=1}^{r} \rho_{i}\left(P_{i}-P_{0}\right)$

for any matrix $P_{0}$ such that $P_{i}-P_{0} \geq 0$. The matrix $P_{0}$ is a slack variable introducing an additional degree of freedom [19]. In the remaining of the paper, $P_{0}$ is chosen to be symmetric. Then, the time derivative of the Lyapunov function is bounded as follows

$$
\begin{aligned}
\dot{V}(e(t)) & \leq e^{T}(t)\left(\Phi_{\hat{\mu} \hat{\mu}}^{T} P_{\hat{\mu}}+P_{\hat{\mu}} \Phi_{\hat{\mu} \hat{\mu}}\right. \\
& \left.+\sum_{i=1}^{r} \rho_{i}\left(P_{i}-P_{0}\right)+\lambda \eta^{2} I+\lambda^{-1} P_{\hat{\mu}} P_{\hat{\mu}}\right) e(t) \\
& \leq e^{T}(t)\left(A_{\hat{\mu}}^{T} P_{\hat{\mu}}+P_{\hat{\mu}} A_{\hat{\mu}}-C^{T} L_{\hat{\mu}}^{T}-L_{\hat{\mu}} C\right. \\
& \left.+\sum_{i=1}^{r} \rho_{i}\left(P_{i}-P_{0}\right)+\lambda \eta^{2} I+\lambda^{-1} P_{\hat{\mu}} P_{\hat{\mu}}\right) e(t)(23)
\end{aligned}
$$

The negativity of $\dot{V}(e(t))$ is ensured if

$$
\begin{gathered}
A_{\hat{\mu}}^{T} P_{\hat{\mu}}+P_{\hat{\mu}} A_{\hat{\mu}}-C^{T} L_{\hat{\mu}}^{T}-L_{\hat{\mu}} C \\
+\sum_{i=1}^{r} \rho_{i}\left(P_{i}-P_{0}\right)+\lambda \eta^{2} I+\lambda^{-1} P_{\hat{\mu}} P_{\hat{\mu}}<0
\end{gathered}
$$

This inequality can be expressed as follows using the Schur complement

$$
M_{\hat{\mu} \hat{\mu}}<0
$$

where $M_{i j}$ is defined by (13). Since the weighting functions satisfy (2), the inequality (25) holds if the LMIs (11) are satisfied, which achieves the proof.

\section{Relaxed LMI formulation of stability conditions of the state estimation error}

Note that the negativity of (25) is largely studied in the literature. An intuitive and first result on stability of T-S systems is the negativity of each term of the sum (25), leading to the result presented above. This result may be very conservative; relaxed stability conditions have been provided using various approaches like Tuan's lemma [31] and Polya's theorem [25]. With the use of Tuan's lemma, inequality (25) holds if the following following inequalities are satisfied

$$
\left\{\begin{array}{l}
M_{i i}<0, i=1, \ldots, r \\
\frac{2}{r-1} M_{i i}+M_{i j}+M_{j i}<0, j \neq i
\end{array}\right.
$$

The power of this result is its significant conservatism reduction without adding slack variables to increase the number of degree of freedom.

Another less restrictive approach is given by simple manipulations of the sums in order to obtain the LMIs [25], [14], in instance, by multiplying by $\sum_{k=1}^{r} \mu_{k}(\hat{x})=1$, one obtains

$$
\left\{\begin{array}{l}
M_{i i}<0, i=1, \ldots, r \\
M_{i i}+M_{i j}+M_{j i}<0, j \neq i \\
M_{i j}+M_{j i}+M_{i k}+M_{k i}+M_{j k}+M_{k j}<0, \\
i \neq j, i \neq k, j \neq k
\end{array}\right.
$$

Note also that most of relaxed stability approaches proposed recently are expressed as LMI with double summation indexes, as the LMI given in (25). Consequently, one of the advantages of using nonquadratic Lyapunov functions is, especially, to find an inequality with a double sum as shown in (25).

\section{GUARANTEED BOUNDED RECONSTRUCTION ERROR}

In the first proposed approach, asymptotic convergence of the state estimation error is sought, based on the (assumed) Lipschitz property of the disturbance-like term. This can only be ensured for some values of the Lipschitz constant (feasibility of the LMIs). In the second approach, the goal is no longer asymptotic convergence, but only convergence in a ball. In that case, the a robust observer designed for T-S systems with UPV does not need the Lipschitz assumption anymore. Thus, the first objective is to provide LMI conditions where the Lipschitz constant $\eta$ is not needed. The second objective is to design a robust observer with respect to modeling uncertainties. For that purpose, a bounded estimation error convergence is proven instead of asymptotic one, by considering ISS.

\section{A. Bounded estimation error}

Let us consider the system (5) and the corresponding proposed observer (6). The state estimation error dynamics is given by (7). Consider also the same nonquadratic Lyapunov function defined in (15) as well as the following assumptions

- A3. The input $u(t)$ is bounded

- A4. The system is input-to-state stable (ISS), i.e. the system state $x(t)$ is bounded for bounded input $u(t)$

- A5. There exists positive scalars $\rho_{i}$ such that the weighting functions satisfy $\mid \dot{\mu}_{i}\left(\hat{x}(t) \mid \leq \rho_{i}\right.$.

These assumptions lead to a bounded perturbation term $\delta(x, \hat{x}, u)$. For the sake of clarity, this term will be noted $\delta(t)$ in the following. One can note that the Lipschitz assumption of the function $f(\hat{x}, x, u)$ is no longer needed. The convergence conditions of the state estimation error are established in the theorem 2.

Definition 1: [26] The system (7) is said to be ISS if there exists a $\mathscr{K} \mathscr{L}$ function $\beta: \mathbb{R}^{n} \times \mathbb{R} \rightarrow \mathbb{R}$ and a $\mathscr{K}$ function 
$\alpha: \mathbb{R} \rightarrow \mathbb{R}$ such that, for each input $\delta(t)$ satisfying $\|\delta(t)\|_{\infty}<$ $\infty$ and each initial condition $e(0) \in \mathbb{R}^{n}$, the trajectory of (7) associated with $e(0)$ and $\delta(t)$ satisfies

$$
\|e(t)\|_{2} \leq \beta\left(\|e(0)\|_{2}, t\right)+\alpha\left(\|\delta(t)\|_{\infty}\right), \forall t
$$

Theorem 2: Under the assumptions A3, A4 and A5, given a scalar $\alpha>0$, if there exists a symmetric matrix $P_{0}$, symmetric matrices $P_{i}$, gain matrices $L_{i}$ and positive scalars $\gamma$ and $c$ solution to the following optimization problem

$$
\min _{P_{0}, P_{i}, L_{i}, \gamma, c} \gamma
$$

under the constraints

$$
\begin{gathered}
P_{0} \geq I \\
P_{i}-P_{0} \geq 0, i=1, \ldots, r \\
\left\{\begin{array}{l}
\Xi_{i i}<0, i=1, \ldots, r \\
\Xi_{i i}+\Xi_{i j}+\Xi_{j i}<0, j \neq i \\
\Xi_{i j}+\Xi_{j i}+\Xi_{i k}+\Xi_{k i}+\Xi_{j k}+\Xi_{k j}<0, \\
i \neq j, i \neq k, j \neq k
\end{array}\right.
\end{gathered}
$$

where $\Xi_{i j}$ is defined by

$$
\begin{gathered}
\Xi_{i j}=\left(\begin{array}{cc}
A_{i}^{T} P_{j}+P_{j} A_{i}-L_{i} C-C^{T} L_{i}^{T}+\sum_{i=1}^{r} \rho_{i}\left(P_{i}-P_{0}\right)+\alpha P_{j} & P_{j} \\
P_{j} & -c I
\end{array}\right) \\
c-\alpha \gamma \leq 0
\end{gathered}
$$

then the error dynamics (7) is ISS with respect to $\delta(t)$ and satisfy the following inequality

$$
\|e(t)\|_{2} \leq \sqrt{\frac{\alpha_{2}}{\alpha_{1}}}\|e(0)\|_{2} e^{-\frac{\alpha}{2} t}+\sqrt{\frac{c}{\alpha \alpha_{1}}}\|\delta(t)\|_{\infty}
$$

The gains $L_{i}$ of the observer are obtained directly and the attenuation level of the transfer from $\delta(t)$ to $e(t)$ is $\sqrt{\frac{c}{\alpha \alpha_{1}}}$.

Proof: Let us consider the nonquadratic Lyapunov function defined by (15). Following the same steps as in (16)-(18), one obtains

$$
\begin{aligned}
\dot{V}(e(t)) & =e^{T}(t)\left(\Phi_{\hat{\mu} \hat{\mu}}^{T} P_{\hat{\mu}}+P_{\hat{\mu}} \Phi_{\hat{\mu} \hat{\mu}}+\dot{P}_{\hat{\mu}}\right) e(t) \\
& +2 e^{T}(t) P_{\hat{\mu}} \delta(t)
\end{aligned}
$$

With (22)-(21), the equality (35) is bounded as follows

$$
\begin{aligned}
\dot{V}(e(t)) \leq & e^{T}(t)\left(\Phi_{\hat{\mu} \hat{\mu}}^{T} P_{\hat{\mu}}+P_{\hat{\mu}} \Phi_{\hat{\mu} \hat{\mu}}+\sum_{i=1}^{r} \rho_{i}\left(P_{i}-P_{0}\right)\right) e(t) \\
& +2 e^{T}(t) P_{\hat{\mu}} \delta(t)
\end{aligned}
$$

From assumptions A3, A4 and A5, the term $\delta(t)$ is bounded. The inequality (36) is equivalent to

$$
\dot{V}(e(t)) \leq e_{a}^{T}(t) \Xi_{\hat{\mu} \hat{\mu}} e_{a}(t)-\alpha e^{T}(t) P_{\hat{\mu}} e(t)+c \delta^{T}(t) \delta(t)
$$

where

$$
\Xi_{\hat{\mu} \hat{\mu}}=\left(\begin{array}{cc}
\Phi_{\hat{\mu} \hat{\mu}}^{T} P_{\hat{\mu}}+P_{\hat{\mu}} \Phi_{\hat{\mu} \hat{\mu}}+\sum_{i=1}^{r} \rho_{i}\left(P_{i}-P_{0}\right)+\alpha P_{\hat{\mu}} & P_{\hat{\mu}} \\
P_{\hat{\mu}} & -c I
\end{array}\right)
$$

and $\alpha, c$ are positive scalars and $e_{a}(t)=\left[\begin{array}{ll}e^{T}(t) & \delta^{T}(t)\end{array}\right]^{T}$. If the inequality (31) holds, the inequality $\Xi_{\hat{\mu} \hat{\mu}}<0$ is also satisfied, then, it follows

$$
\begin{aligned}
\dot{V}(e(t)) & \leq-\alpha e^{T}(t) P_{\hat{\mu}} e(t)+c \delta^{T}(t) \delta(t) \\
& \leq-\alpha V(e(t))+c \delta^{T}(t) \delta(t)
\end{aligned}
$$

Multplying both sides of (40) by $e^{\alpha t}$ and integrating from 0 to $t$, one obtains

$$
V(e(t)) \leq V(0) e^{-\alpha t}+c \int_{0}^{t} e^{-\alpha(t-s)}\|\delta(s)\|_{2}^{2} d s
$$

Due to the fact that the weighting functions $\mu_{i}(\hat{x})$ satisfy (2), then it is easy to derive that, for any $e(t) \in \mathbb{R}^{n}$, it holds

$$
\alpha_{1}\|e(t)\|_{2}^{2} \leq V(e(t)) \leq \alpha_{2}\|e(t)\|_{2}^{2}, \forall e(t) \in \mathbb{R}^{n}
$$

where

$$
\alpha_{1}=\min _{1 \leq i \leq r} \lambda_{\min }\left(P_{i}\right) \quad \alpha_{2}=\max _{1 \leq i \leq r} \lambda_{\max }\left(P_{i}\right)
$$

and where $\lambda_{\min }(M)$ (resp. $\lambda_{\max }(M)$ ) denotes the minimal (resp. maximal) eigenvalue of the matrix $M$. The inequality (41) becomes

$$
\alpha_{1}\|e(t)\|_{2}^{2} \leq \alpha_{2}\|e(0)\|_{2}^{2} e^{-\alpha t}+\frac{c}{\alpha}\|\delta(t)\|_{\infty}^{2}
$$

which leads to

$$
\|e(t)\|_{2}^{2} \leq \frac{\alpha_{2}}{\alpha_{1}}\|e(0)\|_{2}^{2} e^{-\alpha t}+\frac{c}{\alpha \alpha_{1}}\|\delta(t)\|_{\infty}^{2}
$$

Finally, using the square root on equation (45), on obtains

$$
\|e(t)\|_{2} \leq \sqrt{\frac{\alpha_{2}}{\alpha_{1}}}\|e(0)\|_{2} e^{-\frac{\alpha}{2} t}+\sqrt{\frac{c}{\alpha \alpha_{1}}}\|\delta(t)\|_{\infty}
$$

From this equation, it can be concluded that if $\|\delta(t)\|_{\infty}=0$ then $\|e(t)\|_{2} \rightarrow 0$ when $t \rightarrow \infty$. Moreover, in the presence of the perturbation $\delta(t)$, the error $\|e(t)\|_{2}$ is bounded by $\sqrt{\frac{c}{\alpha \alpha_{1}}}\|\delta(t)\|_{\infty}$ at steady state. As a conclusion, the ISS is proven with the inequality (46). The radius of the convergence region $D$ is upper bounded by $\sqrt{\frac{c}{\alpha \alpha_{1}}}\|\delta(t)\|_{\infty}$.

Note that the size of the convergence set $D$ depends on the selected matrices $P_{i}$ and the parameters $\alpha$ and $c$. The set $D$ should be made as small as possible to ensure a good accuracy of convergence. The choice of $\alpha, c$ and $P_{i}$ providing a small set of convergence is not obvious because the problem is nonlinear. In the next, a technique is proposed to transform the nonlinear problem into a linear one leading to LMI constraints. Let us consider the following inequality

$$
\sqrt{\frac{c}{\alpha \alpha_{1}}} \leq \sqrt{\gamma}
$$

where $\gamma$ is a positive scalar to minimize. From (29) and (30), it follows that $\alpha_{1} \geq 1$, then using (47) and for a given $\alpha>0$ the LMI constraint (33) is obtained, which ends the proof.

Remark 1: The second result aims to provide LMI conditions without needing any calculation of the Lipschitz constant. Then, this last has a larger domain of applicability 
compared to the first one. The price to pay is the loss of asymptotic convergence which is replaced by the ISS.

Remark 2: The proposed results are defined by a double sum inequalities represented by

$$
M_{\hat{\mu} \hat{\mu}}<0
$$

This form is adequate to use recent results on conservatism reduction, namely the Polya's theorem. It is easy to derive these conditions (as given in the theorem 2) [14], [25].

Remark 3: Note that the results are easily extended to systems with nonlinear output equation given by

$$
y(t)=\sum_{i=1}^{r} \mu_{i}(x) C_{i} x(t)
$$

\section{B. Robustness with respect to modeling uncertainties}

The observer proposed in the last section guarantees bounded estimation error. It is easy to prove that this observer is robust for some bounded modeling uncertainties. Indeed, the uncertainties can be included in the disturbance-like term obtained from the fact that the weighting functions of the T-S model are unmeasurable. Consider the uncertain system:

$$
\left\{\begin{array}{l}
\dot{x}(t)=\left(A_{\mu}+\Delta A_{\mu}\right) x(t)+\left(B_{\mu}+\Delta B_{\mu}\right) u(t) \\
y(t)=(C+\Delta C) x(t)
\end{array}\right.
$$

with the observer (6), the state estimation error obeys to the differential equation

$$
\dot{e}(t)=\Phi_{\hat{\mu} \hat{\mu}} e(t)+\delta(x, \hat{x}, u)
$$

where $\Phi_{\hat{\mu} \hat{\mu}}=A_{\hat{\mu}}-P_{\hat{\mu}}^{-1} L_{\hat{\mu}} C$ and

$$
\begin{aligned}
\delta(x, \hat{x}, u) & =\left(A_{\mu}-A_{\hat{\mu}}+\Delta A_{\mu}-P_{\hat{\mu}}^{-1} L_{\hat{\mu}} \Delta C\right) x(t) \\
& +\left(B_{\mu}-B_{\hat{\mu}}+\Delta B_{\mu}\right) u(t)
\end{aligned}
$$

Note that the state estimation error (51) has the same form than the one given in (7). All the uncertain terms are included in the disturbance-like term $\delta(x, \hat{x}, u)$. This fact allows to use the result obtained in the last section and hence to prove the ISS from $\delta$ to the state estimation error. The only difference concerns the bound of this perturbation term. Then by minimizing the effect of $\delta(x, \hat{x}, u)$ a robust observer is obtained with respect to some modeling uncertainties. However, note that in the presence of modeling uncertainties, the proposed technique is conservative because it does not take into account the distribution matrices of the uncertain parameters.

Remark 4: The ISS is also preserved if any bounded additive disturbances $\omega(t)$ and $v(t)$ respectively affect the state dynamics and measurement equations. The term $\delta(x, \hat{x}, u)$ can include the disturbances as follows

$$
\delta(x, \hat{x}, u)=\left(A_{\mu}-A_{\hat{\mu}}\right) x+\left(B_{\mu}-B_{\hat{\mu}}\right) u+\omega+P_{\hat{\mu}}^{-1} L_{\hat{\mu}} v
$$

For bounded $\omega(t)$ and $v(t)$, then $\delta(x, \hat{x}, u)$ is still bounded. In addition, the proposed observer can take into account uncertainties on the premise variables.

\section{Simulation EXAMPLE}

Due to space limitation, only the second approach is illustrated and discussed. Let us consider the Rossler chaotic system [16] written as (1) with two sub-models $(r=2)$ defined as follows

$$
\begin{gathered}
A_{1}=\left(\begin{array}{ccc}
-10 & 10 & 0 \\
28 & -1 & -x_{1 \max } \\
0 & x_{1 \max } & -0.37
\end{array}\right), \\
A_{2}=\left(\begin{array}{ccc}
-10 & 10 & 0 \\
28 & -1 & -x_{1 \min } \\
0 & x_{1 \min } & -0.37
\end{array}\right), \\
B_{1}=B_{2}=\left(\begin{array}{c}
0 \\
0 \\
0
\end{array}\right), C=\left(\begin{array}{lll}
0 & 1 & 0 \\
0 & 0 & 1
\end{array}\right)
\end{gathered}
$$

The premise variable is $x_{1}(t)$ which is bounded by $x_{1 \mathrm{~min}}=$ -9.8693 and $x_{1 \max }=13.8164$. The weighting functions are defined by

$$
\begin{aligned}
& \mu_{1}(x(t))=\frac{x_{1}(t)-x_{1 \min }}{x_{1 \max }-x_{1 \min }} \\
& \mu_{2}(x(t))=\frac{x_{1 \max }-x_{1}(t)}{x_{1 \max }-x_{1 \min }}
\end{aligned}
$$

The time derivatives of $\mu_{i}(x), i \in\{1,2\}$ are bounded by $\rho_{1}=$ $\rho_{2}=4.5$. The Lipschitz constant of the term $\delta(t)$ is computed and given by $\eta=173.35$. The approach given in [3] does not provide any solution to this example, since solution can only be obtained for Lipschitz constant smaller than 29.73. The LMIs given in the Theorem 1 of the present paper are also unfeasible for the Lipschitz constant $\eta=173.35$. On the other hand, solving the optimization problem given in the Theorem 2 with SEDUMI (YALMIP), the LMIs are feasible for $\alpha=10$ and the attenuation level of the transfer of $\delta(t)$ towards $e(t)$ is $\sqrt{\gamma}=0.0549$ which is greater than $\sqrt{\frac{c}{\alpha \alpha_{1}}}$. The initial conditions of the system and the observer are $x(0)=\left[\begin{array}{lll}30 & -3 & 10\end{array}\right]^{T}$ and $\hat{x}(0)=\left[\begin{array}{lll}0 & 10 & 40\end{array}\right]^{T}$. The result of the state estimation is depicted on figure 1 .

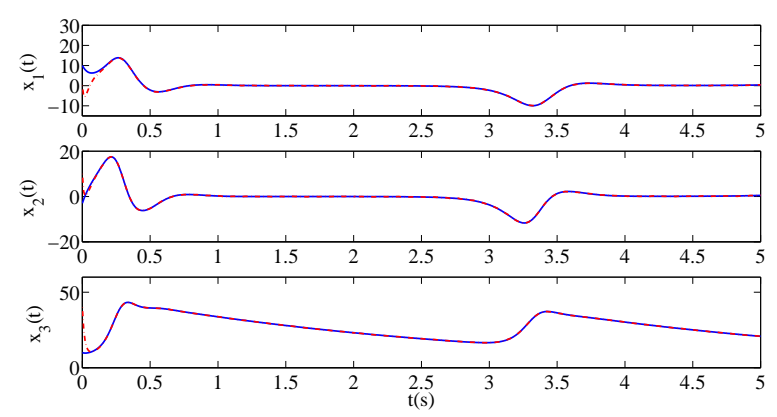

Fig. 1. State variables (blue line) and their estimates (red dashed lines)

A second simulation is performed with a centered measurement noise $v(t)$ in the range -0.33 and 0.33 . This case corresponds to a perturbation term $\delta(t)$ given in equation (53) with $\omega(t)=0$. The norms $\|e(t)\|_{2}$ and $\sqrt{\gamma}\|\delta(t)\|_{\infty}$ are depicted in the figure 2 and illustrates that the norm of the error is always less than $\sqrt{\gamma}\|\delta(t)\|_{\infty}$. 




Fig. 2. Time evolution of $\|e(t)\|$ (red) and $\sqrt{\gamma}\|\delta(t)\|$ (blue) with noised measurement

\section{CONCLUSIONS AND FUTURE WORKS}

In this paper, some advances in observer design for TakagiSugeno systems with UPV are proposed. A focus has been made on some problems, namely, the conservativeness of the existing results on observer design for T-S systems with UPV and the maximal admissible Lipschitz constant allowing to solve the LMI constraints, and the robustness with respect to bounded modeling uncertainties and bounded additive perturbations. The first problem is dealt with by using a nonquadratic Lyapunov function and asymptotic stability conditions are provided. For the second problem, the nonquadratic Lyapunov function and ISS concept are combined. The obtained results are expressed in terms as optimization problems under LMI constraints. For future work, it is interesting to extend the results for both state and unknown input estimation for possible application in fault diagnosis and fault tolerant control of nonlinear systems. An extension of some recent results will be considered for LMI conditions independent from derivative bounds of the weighting functions.

\section{REFERENCES}

[1] A. Akhenak, M. Chadli, J. Ragot, and D. Maquin. Design of sliding mode unknown input observer for uncertain Takagi-Sugeno model. In 15th Mediterranean Conference on Control and Automation, MED'07, Athens, Greece, June 2007.

[2] A. Akhenak, M. Chadli, J. Ragot, and D. Maquin. Fault detection and isolation using sliding mode observer for uncertain Takagi-Sugeno fuzzy model. In 16th Mediterranean Conference on Control and Automation, Ajaccio, France, June 2008.

[3] P. Bergsten and R. Palm. Thau-Luenberger observers for TS fuzzy systems. In 9th IEEE International Conference on Fuzzy Systems, FUZZ IEEE, San Antonio, TX, USA, 2000.

[4] P. Bergsten, R. Palm, and D. Driankov. Fuzzy observers. In IEEE International Fuzzy Systems Conference, Melbourne, Australia, 2001.

[5] P. Bergsten, R. Palm, and D. Driankov. Observers for Takagi-Sugeno fuzzy systems. IEEE Transactions on Systems, Man and Cybernetics - Part B: Cybernetics, 32(1):114-121, 2002.

[6] C. Edwards, S.K. Spurgeon, and R.J. Patton. Sliding mode observers for fault detection and isolation. Automatica, 36(4):541-553, 2000.

[7] H. Hammouri, G. Bornard, and K. Busawon. High gain observer for structured multi-output nonlinear systems. IEEE Transactions on Automatic Control, 55(4):987-992, April 2010.

[8] H. Hammouri and B. Ucchueddu. High gain observers based on immersion technics. In 49th IEEE Conference on Decision and Control, CDC 2010, Atlanta, GA, USA, December 2010.

[9] M. Heemels, J. Daafouz, and G. Millerioux. Observer-based control of discrete-time LPV systems with uncertain parameters. IEEE Transactions on Automatic Control, 55(9):2130-2135, September 2010.
[10] D. Ichalal, B. Marx, D. Maquin, and J. Ragot. On observer design for nonlinear Takagi-Sugeno systems with unmeasurable premise variables. In International Symposium on Advanced Control of Industrial Processes, ADCONIP 2011, Hangzhou, P.R. China, May 2011.

[11] D. Ichalal, B. Marx, J. Ragot, and D. Maquin. Design of observers for Takagi-Sugeno systems with immeasurable premise variables: an $\mathscr{L}_{2}$ approach. In IFAC World Congress, Seoul, Korea, July 2008.

[12] D. Ichalal, B. Marx, J. Ragot, and D. Maquin. An approach for the state estimation of Takagi-Sugeno models and application to sensor fault diagnosis. In 48th IEEE Conference on Decision and Control, CDC'2009, Shanghai, P.R. China, December 2009.

[13] D. Ichalal, B. Marx, J. Ragot, and D. Maquin. State estimation of Takagi-Sugeno systems with unmeasurable premise variables. IET Control Theory and Application, 4(5):897-908, May 2010.

[14] D. Ichalal, B. Marx, J. Ragot, and D. Maquin. New fault tolerant control strategies for nonlinear Takagi-Sugeno systems. International Journal of Applied Mathematics and Computer Science, AMCS, 22(1), January 2012

[15] Zs. Lendek, T.M. Guerra, R. Babuska, and B. De Schutter. Stability Analysis and Nonlinear Observer Design Using Takagi-Sugeno Fuzzy Models, volume 262 of Studies in Fuzziness and Soft Computing. Springer, Germany, 2010.

[16] C. Letellier, S. Elaydi, L. A. Aguirre, and A. Alaoui. Difference equations versus differential equations, a possible equivalence for the Rössler system? Physica D-Nonlinear Phenomena, 195(1-2):29-49, 2004.

[17] B. Marx, D. Koenig, and J. Ragot. Design of observers for Takagi Sugeno descriptor systems with unknown inputs and application to fault diagnosis. IET Control Theory and Application, 1(5):1487-1495, 2007.

[18] G. Millérioux, L. Rosier, G. Bloch, and J. Daafouz. Bounded State Reconstruction Error for LPV Systems With Estimated Parameters. IEEE Transactions on Automatic Control, 49(8):1385-1389, August 2004.

[19] L.A. Mozelli, R.M. Palhares, F.O. Souza, and E.M.A.M. Mendes. Reducing conservativeness in recent stability conditions of TS fuzzy systems. Automatica, 45(6):1580-1583, 2009.

[20] R. Palm and P. Bergsten. Sliding mode observers for Takagi-Sugeno fuzzy systems. 9th IEEE International Conference on Fuzzy Systems, FUZZ IEEE, San Antonio, TX, USA, 2000.

[21] R.J. Patton, J. Chen, and C.J. Lopez-Toribio. Fuzzy observers for nonlinear dynamic systems fault diagnosis. In 37th IEEE Conference on Decision and Control, Tampa, Florida USA, 1998.

[22] A.M. Pertew, H.J. Marquez, and Q. Zhao. $\mathrm{H}_{\infty}$ observer design for Lipschitz nonlinear systems. IEEE Transactions on Automatic Control, 51(7):1211-1216, July 2006.

[23] S. Raghavan and J. K. Hedrick. Observer design for a class of nonlinear systems. International Journal of Control, 59(2):515-528, 1994.

[24] R. Rajamani. Observers for Lipschitz nonlinear systems. IEEE Transactions on Automatic Control, 43(3):397-401, March 1998.

[25] A. Sala and C. Ariño. Asymptotically necessary and sufficient conditions for stability and performance in fuzzy control: Applications of Polya's theorem. Fuzzy Sets and Systems, 158(24):2671-2686, December 2007.

[26] E.D. Sontag. On the input-to-state stability property. Systems \& Control Letters, 24:351-359, 1995.

[27] T. Takagi and M. Sugeno. Fuzzy identification of systems and its applications to modeling and control. IEEE Transactions on Systems, Man and Cybernetics, 15(1):116-132, 1985.

[28] K. Tanaka, T. Hori, and H.O. Wang. A multiple Lyapunov function approach to stabilization of fuzzy control systems. IEEE Transactions on Fuzzy Systems, 11(4):582-589, August 2003.

[29] K. Tanaka and H.O. Wang. Fuzzy Control Systems Design and Analysis: A Linear Matrix Inequality Approach. John Wiley and Sons, 2001.

[30] F.E. Thau. Observing the state of non-linear dynamic systems. International Journal of Control, 18(3):471-479, 1973.

[31] H.D. Tuan, P. Apkarian, T. Narikiyo, and Y. Yamamoto. Parameterized linear matrix inequality techniques in fuzzy control system design. IEEE Transactions on Fuzzy Systems, 9(2):324-332, 2001.

[32] J. Yoneyama. $H_{\infty}$ filtering for fuzzy systems with immeasurable premise variables: an uncertain system approach. Fuzzy Sets and Systems, 160(12):1738-1748, 2009. 